This is a post-peer-review, pre-copyedit version of:

Masotti N., Bagassi S., De Crescenzio F. (2016) Augmented Reality for the Control Tower: The RETINA Concept. In: De Paolis L., Mongelli A. (eds) Augmented Reality, Virtual Reality, and Computer Graphics. AVR 2016. Lecture Notes in Computer Science, vol 9768. Springer, Cham.

The final authenticated version is available online at: https://doi.org/10.1007/978-3319-40621-3 32

This version is subjected to Springer Nature terms for reuse that can be found at: https://www.springer.com/gb/openaccess/authors-rights/aam-terms-v1 


\title{
Augmented Reality for the Control Tower: The RETINA Concept
}

\author{
Nicola Masotti, Sara Bagassi, Francesca De Crescenzio \\ Department of Industrial Engineering \\ University of Bologna \\ Bologna, Italy \\ \{nicola.masotti, sara.bagassi, \\ francesca.decrescenzio\} dunibo.it
}

\begin{abstract}
The SESAR (Single European Sky Air Traffic Management Research) Joint Undertaking has recently granted the Resilient Synthetic Vision for Advanced Control Tower Air Navigation Service Provision project within the framework of the H2020 research on High Performing Airport Operations. Hereafter, we describe the project motivations, the objectives, the proposed methodology and the expected impacts, i.e. the consequences of using virtual/augmented reality technologies in the control tower.
\end{abstract}

Keywords: Air·Traffic $\cdot$ Control·Airport·Tower $\cdot$ Virtual $\cdot$ Augmented $\cdot$ Reality Synthetic $\cdot$ Vision

\section{Overview}

In the latest years, many of the technological advancements designed to improve the airport operational safety have come in the form of innovative visualization tools for tower controllers. Surface Movement Guidance and Control System (SMGCS) based solutions, such as movement maps, conformance monitoring, and conflict detection are a few examples of these tools. However, there is a paradox in developing these tools to increase the control-tower air traffic controller's situational awareness. By creating additional computer displays that show the runway and taxiway layout, aircrafts and vehicles position, and detect actual and foreseen conflicts, the controller's vision is pulled away from the out of the window view and his or her 'head-down' time is increased'. This reduces their situational awareness by forcing them mentally to repeatedly switch between these two ways of interpreting the working environment. In order to be able to address this paradox, Lloyd Hitchcock introduced the idea of using AR in the control tower when the Augmented Reality technology was still in the very early stages of its industrialization. At that time, no prototype construction was attempted and little was published, though many recall Mr. Hitchcock speculating on several methods that could

1 The 'head-down' time is the time spent by the air traffic controller looking at his/her desk equipment or managing flight strips. 
aid tower controllers fulfilling their tasks [1-5]. For instance, he suggested that AR displays could provide air traffic controllers with useful status information, such as aircraft identification, barometer settings, wind conditions and runway or gate assignments. More recent studies suggest that other spatially conformal information, such as flight tags, warnings, shapes and layouts can also be presented on AR displays [2, 612]. Indeed, the scientific community has already performed many experiments with by now out-dated hardware $[13,14]$.

The Resilient Synthetic Vision for Advanced Control Tower Air Navigation Service Provision (RETINA) project is one of the selected Single European Sky ATM Research (SESAR) projects on High Performing Airport Operations that will investigate the potential and applicability of Virtual/Augmented Reality (V/AR) technologies for the provision of Air Traffic Control (ATC) service by the airport control tower. The project will assess whether those concepts that stand behind tools such as Head-Mounted Displays (HUDs), Enhanced Vision Systems (EVSs) and Synthetic Vision Systems (SVS) can be transferred to ATC with relatively low effort and substantial benefits for controllers' Situational Awareness (SA). In doing so, the project Consortium (Fig. 1) will investigate two different augmented reality systems: Conformal-Head-Up Displays (CHUDs) - which, potentially, can be made to coincide with the tower windows - and See-Through Head-Mounted Displays (ST-HMD). This will be done by means of outoff-the-shelf AR hardware components. A dissimilar third tool, i.e. a virtual reality based Table-Top interface, will be conceived as well, since the upper view is the easiest way to visualize the airport digital model (Fig. 2).

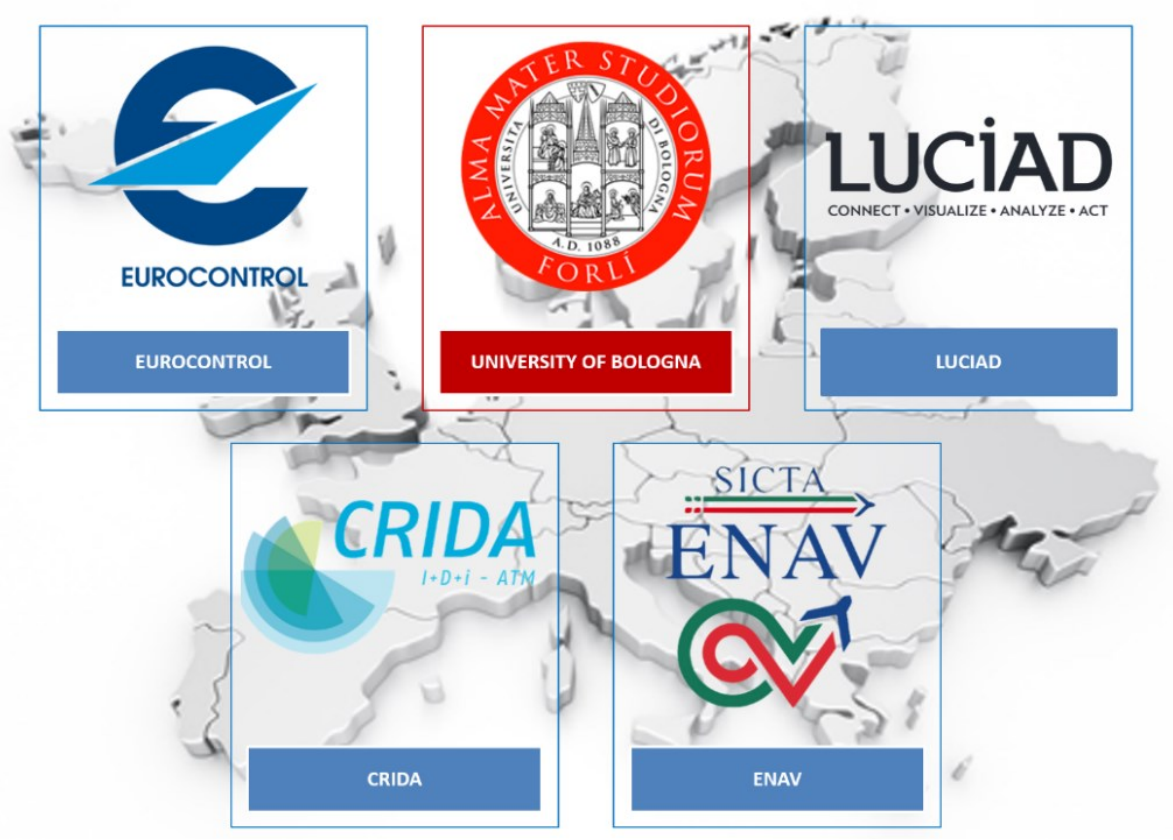

Fig. 1. The RETINA project consortium. 
Because the focus of the project will be the placement of information over the actual window view, the relationship between conformal (registered) information and the user's perspective is a major issue. This subject has been widely studied in other fields such as cultural heritage, entertainment and virtual interaction. For instance, several studies have demonstrated that, at any time, the conceived AR environment must be aware of the user's eyes position with respect to the Virtual/Augmented Reality (V/AR) screens [15-17]. This is mandatory in order to generate (render) the best AR content for each eye and achieve the best registration possible. Within the RETINA project this concept will be implemented and demonstrated by means of non-intrusive, out-of-theshelf, body-tracking sensors (e.g. MicrosoftTM KinectTM) or taking advantage of the tracking capabilities of certain HMDs (e.g. MicrosoftTM HoloLensTM). Thus, the AR screens would know where the controller is and where s/he is looking, allowing the interface to present the most beneficial information without adding needless clutter. Cues to critical situations that take place outside of the controllers view can also be placed in controllers' peripheral vision, to draw their attention in that direction. Overall, the information that is currently displayed on the head-down computer screens (flight tags, runway layout, intrusion warnings) could be displayed on either the see-through glasses or the head-up displays, therefore superimposed to the controller's line of sight.

As a common database between the V/AR systems, a three-dimensional Aerodrome Traffic Zone (ATZ) model will be developed and implemented, providing precise positioning for simulated aerial and terrestrial objects. Multiple simulated or recorded data sources such as Airport Surveillance Radar, Surface Movement Radar or other groundbased sensors (e.g. video or infrared cameras) will provide the displayed information (Fig. 2). In this respect, the RETINA project foresees a technology transfer between remote and on-site tower operations. Indeed, a proper 2D camera distribution within the simulated environment can provide reliable data regarding the positioning, speed, speed direction and size of ground-based objects. This is particularly convenient for smaller airports, where installing an Advanced Surface Movement Guidance and Control System (A-SMGCS) is deemed too much expensive. In larger airports, such sensors could still be useful to cover distant and blind spots, improving the controllers' SA of the surrounding area.

Other information that can be displayed to the controller includes SWIM (System Wide Information Management) related data, such as weather conditions, wind direction and speed, wind shear and wake vortexes visualization. Within the SESAR, the SWIM concept is the enabler for ensuring the delivery of the proper information, with the required quality, to the appropriate person at the right time [18]. RETINA will look at SWIM standards and services that can support the need for information of the planned V/AR tower tools. For example, RETINA will investigate how SWIM services can consume and visualize meteorological data using the data exchange format WXXM, one of the AIRM-based data model standards. Weather related information (such as wake vortexes, wind and wind shears) information could be used to optimize separations between approaching and departing aircrafts, leveraging weather related phenomena in a similar manner to what has been done in the SESAR Operational Service and Environment Definition (OSED) 06.08.01 (Time Based Separation) [19, 20]. 


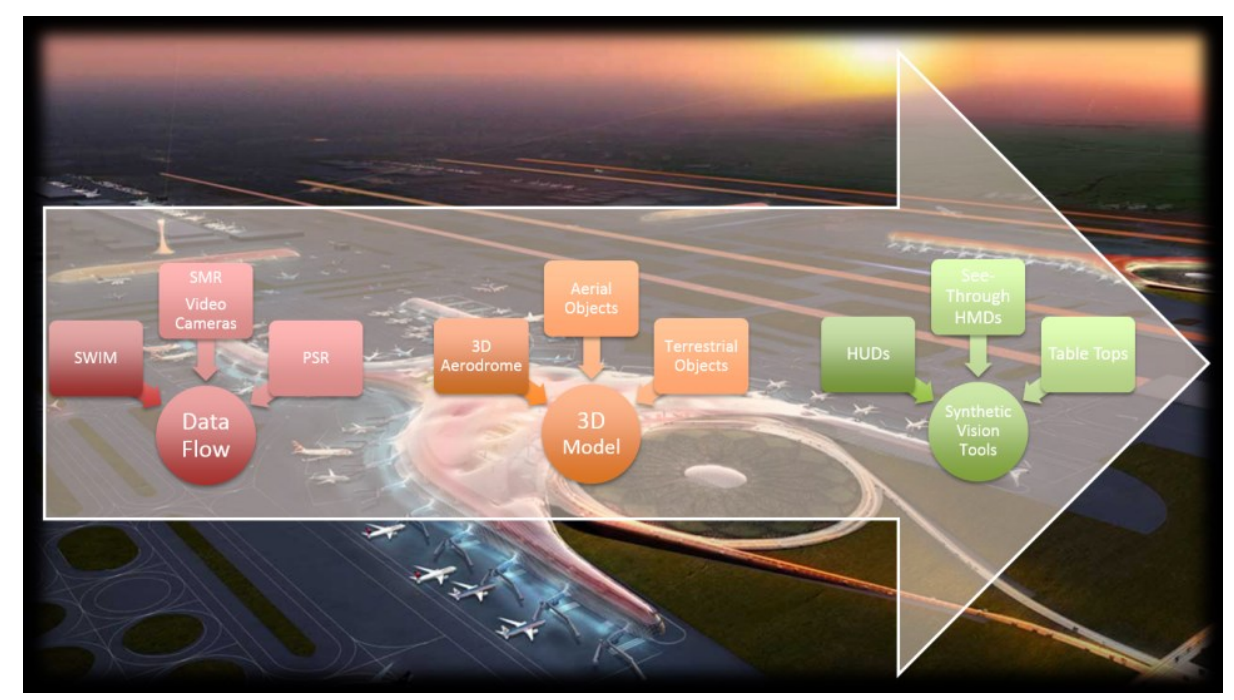

Fig. 2. The overall RETINA Concept.

This adds to the SWIM's overall objective of achieving global Air Traffic Management (ATM) interoperability and standardization.

Finally, the RETINA project will investigate the impact of the newly conceived tools on the control tower traffic management procedures. For instance, in low visibility or bad weather conditions, ad hoc Low Visibility Procedures (LVP) must apply. In many airports, this entails the use of a Surface Movement Radar (SMR), which provides only primary positioning for the ground traffic (without any identification support system). Moreover, depending on the airport layout, Low Visibility Procedures might include constraints, such as such as taxiways that cannot be used, block spacing, limitation in start-up and pushback operations, runway closure or use of a predefined runway. In this context, the use V/ARTT could possibly reduce restraints, producing benefits in terms of safety and capacity. For instance, when relying on Synthetic Vision (SV), an exclusive use of taxiway blocks should not be necessary - i.e. an aircraft could use a segment of a taxiway before the preceding aircrafts has left such segment. Whenever a new technology is used, ATC procedures must change accordingly. Therefore, the RETINA project will define when, why and how controllers will make use of augmented visual observation in order to manage the aerodrome traffic. Ad hoc recovery procedures will be proposed and validated, in order to demonstrate the real world applicability of the proposed solutions. Indeed, in case of a sudden SV failure, controllers must be able to return to standard Low Visibility Procedure in a quick manner, with no real threat to safety. 


\section{Methodology}

In Air Traffic Control, operators must deal with easy tasks and familiar events, as well as with unfamiliar, time consuming and unexpected events. Besides talking to pilots, controllers need to extract information from the PVD, check weather, consult Flight Strips (FS), elaborate long term strategies, detect potential conflicts, make tactical decisions, coordinate with each-other and look out of the tower window (if any) $[21,22]$. In addition, controllers need to balance cognitive resources and carefully timetable actions $[21,22]$. Under these circumstances, human-computer interaction designers cannot only focus on the user but must consider the complexity of the work domain. Within the RETINA project, the interface design will draw from the Ecological Interface Design (EID) approach.

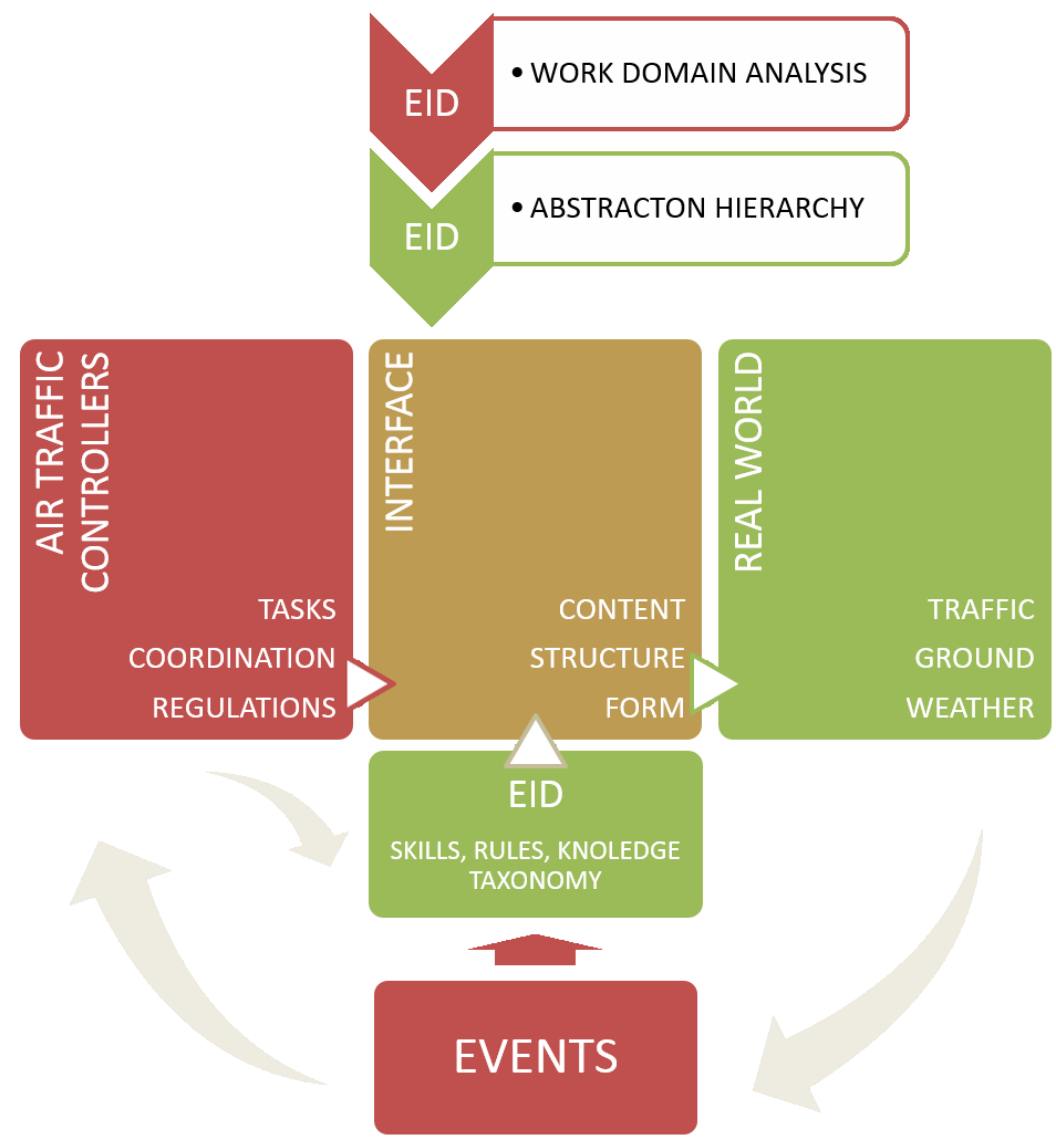

Fig. 3. The EID theoretical framework applied to the control-tower work domain. 
EID is a theoretical framework for designing human-machine interfaces in complex, real-time and dynamic systems. This methodology differs from User-Cantered Design (UCD) insofar it focuses on the analysis of the work domain (a.k.a. Work Domain Analysis - WDA) rather than on the end-user or his/her specific tasks. EID attempts to provide the operators with the necessary tools and information to become active problem solvers as opposed to passive monitors, particularly during the development of unforeseen events [23]. Interfaces designed following the EID approach aim to decrease the mental workload when dealing with unfamiliar and unanticipated events, which are attributed to increased psychological pressure [23]. Doing so, EID makes use of two theoretical pillars from cognitive engineering research: the Abstraction Hierarchy (AH) and the Skills, Rules, Knowledge (SRK) taxonomy.

The Abstraction Hierarchy $(\mathrm{AH})$ is a 5-level functional decomposition used for modelling the work environment (a.k.a. the work domain). In the EID framework, the AH is used to determine what kinds of information should be displayed on the system interface and how the information should be arranged. In doing so the designers attempts to make constraints and complex relationships in the work environment perceptually evident (e.g. visible, audible) to the end user (i.e. the air traffic controller) in order to free up cognitive resources that might support efficient problem solving [5]. As an example, the reader can easily refer to the use of tunnels or highways-in-the-sky for aircraft governance.

The Skills, Rules, Knowledge (SRK) framework (a.k.a. SRK taxonomy) defines three types of behaviour or psychological processes which are present in the operator information processing. The three categories essentially describe three possible ways in which information can be extracted and understood from a human-machine interface [6]. The categories can be weighted according to the user needs. For example, by supporting skill and rule based behaviour in familiar tasks, more cognitive resources may be devoted to knowledge-based behaviour, which are important for managing unanticipated events.

\section{Expected Impacts.}

In 2014, within the European Civil Aviation Conference Area (ECACA), an average delay per flight of 9.7 minutes was developed [24]. Further analysis of the rationale behind the delay show that 0.51 min were due to weather, mainly strong wind, snow and low visibility conditions, whilst $0.96 \mathrm{~min}$ were due to restrictions at the departing or arrival airport, including the typical LVP restrictions defined in section 1 [24]. Also, please notice that these data do not account for cancelled or redirected flights.

If the RETINA concept will ever become operative the proposed solutions will provide concerned actors with high-quality 4D information (position, height and speed over time) in any operational condition (traffic, weather, airport complexity, etc.). Thus the resilience and efficacy of the control tower IT system will be improved as well as the controllers' SA. This will allow Instrument Landing System (ILS) or SV equipped 
aircrafts to seamlessly operate under any visibility condition at synthetic vision equipped airports.

Complex airports will benefit from the implementation of the RETINA concept by preserving airport capacity in all weather conditions, even when LVP apply. This will result in financial savings for carriers and larger incomes for Air Navigation Service Providers (ANSP). In addition, nearby airports will not face the risk of saturation. With fewer delay, a reduction of the environmental impact of flights in terms of fuel burnt, emissions, $\mathrm{CO}_{2}$, etc. will be achieved.

New advancements in the design of a camera-based tracking system will benefit smaller airports by enabling a ground control system through limited investments with respect to those required for a conventional A-SMGCS system. Such implementation will allow extending the provision of cost-effective Air Traffic Services (ATS) to those airports where only the Aerodrome Traffic Information Service (AFIS) is provided (either remotely or locally operated).

Definitely, the RETINA concept could highly contribute to establish a satisfactory level of safety for smaller airports, where traffic volume is simply too low to pay back for the initial investment in a SMR equipment. Side effects, such as the increase of traffic volume at smaller or peripheral airports due to a better level of quality of service must not be neglected. Passengers and couriers could use small aircrafts on a more frequent basis, with positive social impact on the community living in the airport surrounding. Consequently, the number of operations would increase and more airport equipage may be needed.
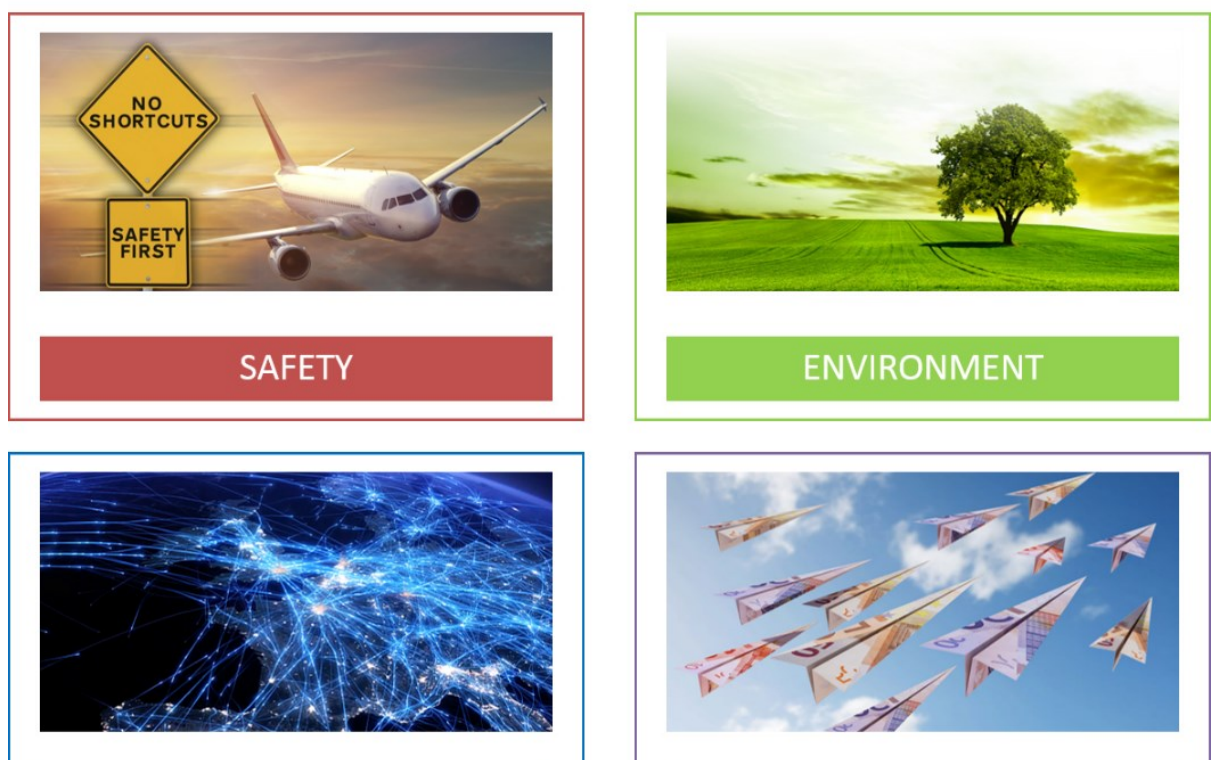

EFFICIENCY

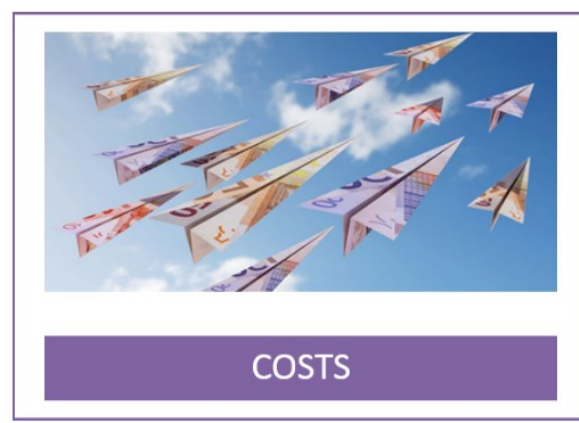

Fig. 4. Areas of interest impacted by the RETINA concept. 
The project will also exploit the SWIM concept allowing for a cost effective standardization and better re-use of data sets and services between the control tower IT systems. With no need for duplicates, significant savings for all ANSP will be achieved.

Overall, the RETINA project is expected to push the Technology Readiness Level (TRL) for V/AR technologies in the control tower from 1 to 2 and consolidate the leading role of European companies (ANSP and industries) into the field of air navigation.

\section{References}

1. Reisman, R., Brown, D.: Design of Augmented Reality Tools for Air Traffic Control Towers. In: Proceedings of 6th AIAA Aviation Technology, Integration and Operations Conference (ATIO). American Institute of Aeronautics and Astronautics, Wichita, KS (2006).

2. Stephen R. Ellis, Bernard D. Adelstein, Ronald J. Reisman, Joelle R. Schmidt-Ott, Jonathan Gips, Jimm Krozel: Augmented Reality in a Simulated Tower Environment: Effect of Field of View on Aircraft Detection. NASA Ames Research Center Technical Reports (2002).

3. Ronald J. Reisman, David M. Brown: Augmented Reality Tower Technology Assessment. NASA Ames Research Center (2010).

4. Peterson, S.: Very large format stereoscopic head-up display for the airport tower. In: In Proceedings of the Virtual Images Seminar, number 16. CNRS/Renault (2007).

5. Ellis, S.R.: Towards Determination of Visual Requirements for Augmented Reality Displays and Virtual Environments for the Airport Tower. In: Virtual Media for Military Applications RTO-MP-HFM-136. pp. 31-1-31-10. RTO, Neuilly-sur-Seine, France (2006).

6. Herman Rediess: An augmented reality pilot display for airport operations under low and zero visibility conditions. In: Guidance, Navigation, and Control Conference. American Institute of Aeronautics and Astronautics (1997).

7. Ruffner, J.W., Deaver, D.M., Henry, D.J.: Requirements analysis for an air traffic control tower surface surveillance enhanced vision system. Presented at the SPIE-The International Society for Optical Engineering (2003).

8. N. Fürstenau: Virtual Tower. 5th ATM R\&D Symposium., Braunschweig (2005).

9. Azuma, R.: A Survey of Augmented Reality. In: Presence: Teleoperators and Virtual Environments 6. pp. 355-385 (1997).

10. Reisman, R.J., Feiner, S.K., Brown, D.M.: Augmented Reality Tower Technology Flight Test. In: International Conference on Human-Computer Interaction in Aerospace (HCIAero)., Santa Clara, CA (2014).

11. De Crescenzio F., Bagassi S., Fantini M., Lucchi F.: Virtual Reality based HUD (Head Up Display) to simulate 3D conformal symbols in the design of future cockpits. Presented at the Council of European Aerospace Societies, Venice, Italy (24-28 October, 2).

12. Sara Bagassi, Francesca De Crescenzio, Francesca Lucchi, Franco Persiani: Innovation in Man Machine Interfaces: Use of 3D Conformal Symbols in the Design of future HUDs (Head Up Displays). Presented at the 28th International Congress of The Aeronautical Sciences , Brisbane, Australia September 23 (2012).

13. Reisman, R.J., Ellis, S.R.: Air Traffic Control Tower Augmented Reality Field Study. In: ACM SIGGRAPH Posters. ACM, New York, NY, USA (2005).

14. Reisman, R., Brown, D.: Design of Augmented Reality Tools for Air Traffic Control Towers. In: Proceedings of 6th AIAA Aviation Technology, Integration and Operations Conference (ATIO). American Institute of Aeronautics and Astronautics, Wichita, KS (2006).

15. Solari, F., Chessa, M., Garibotti, M., Sabatini, S.P.: Natural perception in dynamic stereoscopic augmented reality environments. Displays. 34, 142 - 152 (2013). 
16. Nicola Masotti, Franco Persiani: Gaze-Coupled Perspective for Enhanced Human-Machine Interfaces in Aeronautics. Presented at the Conferences in Air Transport \& Operations, Delft University of Technology, Delft, The Netherlands. July 20 (2015).

17. Rolland, J.P., Baillot, Y., Goon, A.A.: A Survey of Tracking Technology for Virtual Environments. Fundamentals of Wearable Computers and Augmented Reality. 67-112 (chap. 3) (2001).

18. SESAR JU: System Wide Information Management (SWIM), http://www.sesarju.eu/sesarsolutions/swim.

19. SESAR JU: Time Based Separation, http://www.sesarju.eu/sesar-solutions/airport-integration-and-throughput/time-based-separation.

20. Matayoshi, N.: Reduced Wake Vortex Separation Using Weather Information. In: Air Traffic Management and Systems. pp. 49-68. Springer Japan (2014).

21. Masotti, N., Persiani, F.: On the history and prospects of three-dimensional human-computer interfaces for the provision of air traffic control services. CEAS Aeronaut. J. 1-18 (2016).

22. Harper, R.H.R., Hughes, J.A.: "What a f-ing system! Send 'em all to the same place and then expect us to stop 'em hitting": Making Technology Work in Air Traffic Control. In: Button, G. (ed.) Technology in Working Order: Studies of Work, Interaction, and Technology. pp. 127-144. Routledge, London (1993).

23. Vicente, Kim J., Jens Rasmussen: Ecological interface design: Theoretical foundations. Syst. Man Cybern. IEEE Trans. 22, 589-606 (1992).

24. EUROCONTROL: All-Causes Delay and Cancellations to Air Transport in Europe, https://www.eurocontrol.int/publications/coda-digest-annual-2014. 